\title{
Strain-induced stabilization of new magnetic spinel structures in epitaxial oxide heterostructures
}

\author{
F. Rigato ${ }^{a}$, S. Estradé ${ }^{b}$, J. Arbiol ${ }^{b, c}$, \\ F. Peiró ${ }^{b}$, U. Lüders ${ }^{d}$, X. Martí ${ }^{a}$, F. Sánchez ${ }^{a}$, J. Fontcuberta ${ }^{a}{ }^{a} *$ \\ ${ }^{a}$ Institut de Ciència de Materials de Barcelona-CSIC, Campus UAB, E-08193 Bellaterra, Spain \\ ${ }^{\mathrm{b}}$ EME/CeRMAE/IN2UB, Departament d'Electrònica, Universitat de Barcelona, C/Martí Franquès 1, E-08028 Barcelona, Spain \\ ${ }^{\mathrm{c}}$ TEM-MAT, Serveis Cientificotècnics, Universitat de Barcelona, C/Solé i Sabarís 1-3, E-08028 Barcelona, Spain \\ ${ }^{\mathrm{d}}$ LNMH ONERA-CNRS, BP 4025, 31055 Toulouse, Cedex 4, France
}

\begin{abstract}
We report here on the growth of $\mathrm{NiFe}_{2} \mathrm{O}_{4}$ epitaxial thin films of different thickness $(3 \mathrm{~nm} \leq t \leq 32 \mathrm{~nm})$ on single crystalline substrates having spinel $\left(\mathrm{MgAl}_{2} \mathrm{O}_{4}\right)$ or perovskite $\left(\mathrm{SrTiO}_{3}\right)$ structure. Ultrathin films, grown on any of those substrates, display a huge enhancement of the saturation magnetization: we will show that partial cationic inversion may account for this enhancement, although we will argue that suppression of antiparallel collinear spin alignment due to size-effects cannot be excluded. Besides, for thicker films, the magnetization of films on MAO is found to be similar to that of bulk ferrite; in contrast, the magnetization of films on STO is substantially lower than bulk. We discuss on the possible mechanisms leading to this remarkable difference of magnetization.
\end{abstract}

(C) 2007 Elsevier B.V. All rights reserved.

Keywords: Spinel thin films; Ferrites; Epitaxial spinels; Epitaxial strain, Magnetization

\section{Introduction}

Bulk spinel ferrites have been deeply investigated due to their relevance in many technological markets, such as high frequency devices. In the recent years, ferrimagnetic spinel-oxide thin films are also being considered for more advanced applications, such as spintronics. Therefore a detailed understanding of the relationship between film structure and microstructure, their thickness dependence and their correlation with functional parameters (magnetic, electric, etc.) is definitely needed. Moreover, spinel ferrites are nowadays integrated in complex heterostructures. Examples include multilayers formed by oxides with spinel $\left(\mathrm{AB}_{2} \mathrm{O}_{4}\right)$ and perovskite $\left(\mathrm{ABO}_{3}\right)$ structures, used in spintronic devices, such as spin filters [1,2], or multiferroic nanocomposites [3]. Dissimilar structures lead to structural mismatch and thus strained regions are formed at interfaces. The strain field can be relevant to couple some functional properties of each compound but it can also be detrimental for some applications, as it may induce electronic phase separation as reported for strained manganites [4].

\footnotetext{
* Corresponding author.

E-mail address: fontcuberta@icmab.es (J. Fontcuberta).
}

In addition, a detailed understanding of the growth mechanisms of these ferrimagnetic spinel films is fundamental for their utilization, because the growth conditions may introduce new degrees of freedom in tailoring the film properties [1]. It is quite remarkable that the magnetic response of thin films, in general, is very different from the bulk behaviour. It has been found, for instance, that the saturation magnetization of films relatively thick can be substantially lower than the bulk value and this anomalous behavior has been attributed to a large lattice film/substrate mismatch or to the presence of anti-phase boundaries (APB) [5,6]. Interestingly enough, however, the saturation magnetization of ultrathin films of $\mathrm{NiFe}_{2} \mathrm{O}_{4}$ has been found to be substantially larger than bulk magnetization [7]. This unexpected result has been attributed to the out-of-equilibrium atomic distribution resulting from growth conditions $[7,8]$ although definite explanation remains to be settled.

To improve the understanding of the growth conditions influence on the film properties, a systematic growth of $\mathrm{NiFe}_{2} \mathrm{O}_{4}$-NFO films by RF magnetron sputtering has been performed on two different substrates $\left(\mathrm{SrTiO}_{3}-\mathrm{STO}\right.$ and $\mathrm{MgAl}_{2} \mathrm{O}_{4}-\mathrm{MAO}$ ), focusing on the thickness dependence of the magnetic behavior. We note that whereas MAO and NFO are isostructural, this is not the case of NFO and STO, which largely differ in their atomic arrangement. Here we will report data 
on films of thicknesses $t(3 \mathrm{~nm} \leq t \leq \leq 32 \mathrm{~nm})$. We will show that thinner films, irrespectively on the substrate used, display a saturation magnetization $\left(M_{\mathrm{S}}\right)$ remarkably larger than the bulk value; the thickest films, on the contrary, display a magnetization that depends on the substrate: whereas on MAO substrates $M_{\mathrm{S}}$ displays bulk-like values, this is not the case of films grown on STO, where $M_{\mathrm{S}}$ is considerably lower. We will discuss on the origin of these effects; we will argue that although partial inversion of the NFO spinel structure could describe the observed behavior in the thinner films, size-effects can also significantly contribute to this effect; for thicker films the different behavior observed for films grown on STO and MAO casts some questions on the role of anti-phase boundaries.

\section{Experimental}

NFO has a spinel structure with a bulk cell parameter $a_{\mathrm{NFO}} \approx 0.8339 \mathrm{~nm}$; two substrate materials were employed: a spinel (MAO) and a perovskite (STO), both oriented along the [0 001 l] direction, having cell parameters of $a_{\mathrm{MAO}}=0.8083 \mathrm{~nm}$ and $a_{\mathrm{STO}}=0.3905 \mathrm{~nm}$, respectively. Films on MAO and STO were grown, simultaneously, by RF magnetron sputtering, using a stoichiometric target positioned at $60 \mathrm{~mm}$ from the substrate surface in on-axis configuration, and operating in a mixed atmosphere of $\mathrm{O}_{2}$ and Ar, with a 1:10 gas flux ratio. During the processes the total pressure inside the deposition chamber was $33.3 \mathrm{~Pa}(250 \mathrm{mTorr})$ and the substrate temperature was kept at $600{ }^{\circ} \mathrm{C}$.

To get information about the different phases, the out-ofplane texture and the out-of-plane parameter $c$ of the films, X-ray diffraction (XRD) $\theta-2 \theta$ scans were made using a Siemens D-5000 diffractometer and $\mathrm{Cu} \mathrm{K} \alpha$ radiation. The sample thickness was calculated by X-ray reflectometry (XRR), correlating the position of the Kiessig fringes to their order; the thickness of films thinner than $10 \mathrm{~nm}$ was determined by using the calibrated growth rate and controlling growth time. The growth rate was found very similar for NFO films on STO and MAO: $\sim 0.27 \mathrm{~nm} / \mathrm{min}$ and $\sim 0.25 \mathrm{~nm} / \mathrm{min}$, respectively. Films with 14 different thicknesses were grown by varying the deposition time. Here we show, as illustrative examples, data of films of thicknesses $t \approx 2.6,16.9$ and $31.5 \mathrm{~nm}$ on STO and $t \approx 5.0,13.4$ and $29.1 \mathrm{~nm}$ on MAO.

TEM observations were carried out in a Philips CM30 microscope operated at $300 \mathrm{kV}$. For plane view examination, the samples were flat polished by the substrate side up to $50 \mu \mathrm{m}$; the polishing was followed by a dimpling up to $20 \mu \mathrm{m}$ and a final $\mathrm{Ar}^{+}$bombardment at $V=5 \mathrm{kV}$ with an incident angle of $7^{\circ}$, using a PIPS-Gatan equipment. During the whole process, the sample surface was protected in order to prevent layer damage or material redeposition.

Magnetic characterization was performed using a Quantum Design superconducting quantum interference device (SQUID) at $10 \mathrm{~K}$, with external field applied parallel to the sample surface. The diamagnetic contribution to the susceptibility $\left(\chi_{\mathrm{d}}\right)$ results from the sum of the contributions of the substrate and of the sample holder used to perform the measurement; notice that any differential high-field susceptibility originated by the sample will also be included in this term. It was determined by linear regression of the high field region $(40 \mathrm{kOe}<H<50 \mathrm{kOe})$ of the magnetization curves. There is not a significant dependence of the susceptibility on the film thickness: the average value is $\chi_{\mathrm{d}}=(-5.5 \pm 0.9) \times 10^{-7} \mathrm{emu} \mathrm{Oe}^{-1} \mathrm{~cm}^{-3}$ in the case of STO and $\chi_{\mathrm{d}}=(-1.0 \pm 0.1) \times 10^{-6} \mathrm{emu} \mathrm{Oe}^{-1} \mathrm{~cm}^{-3}$ for MAO. Both values are, within the experimental accuracy, coincident with those obtained when measuring the bare substrate: they were subsequently subtracted from the hysteresis loop data.

\section{Results}

Patterns in Fig. 1a and $\mathrm{b}$ show the $\theta-2 \theta$ scans performed on films deposited respectively over MAO and STO substrates. Only data for $13.4 \mathrm{~nm}$ and $29.1 \mathrm{~nm}$ films on MAO and $16.9 \mathrm{~nm}$ and $31.5 \mathrm{~nm}$ films on STO are shown as for films with thickness below $5 \mathrm{~nm}$ the diffracted intensity is exceedingly low and not visible in the X-ray scans. Shown in Fig. 1 are the dif-
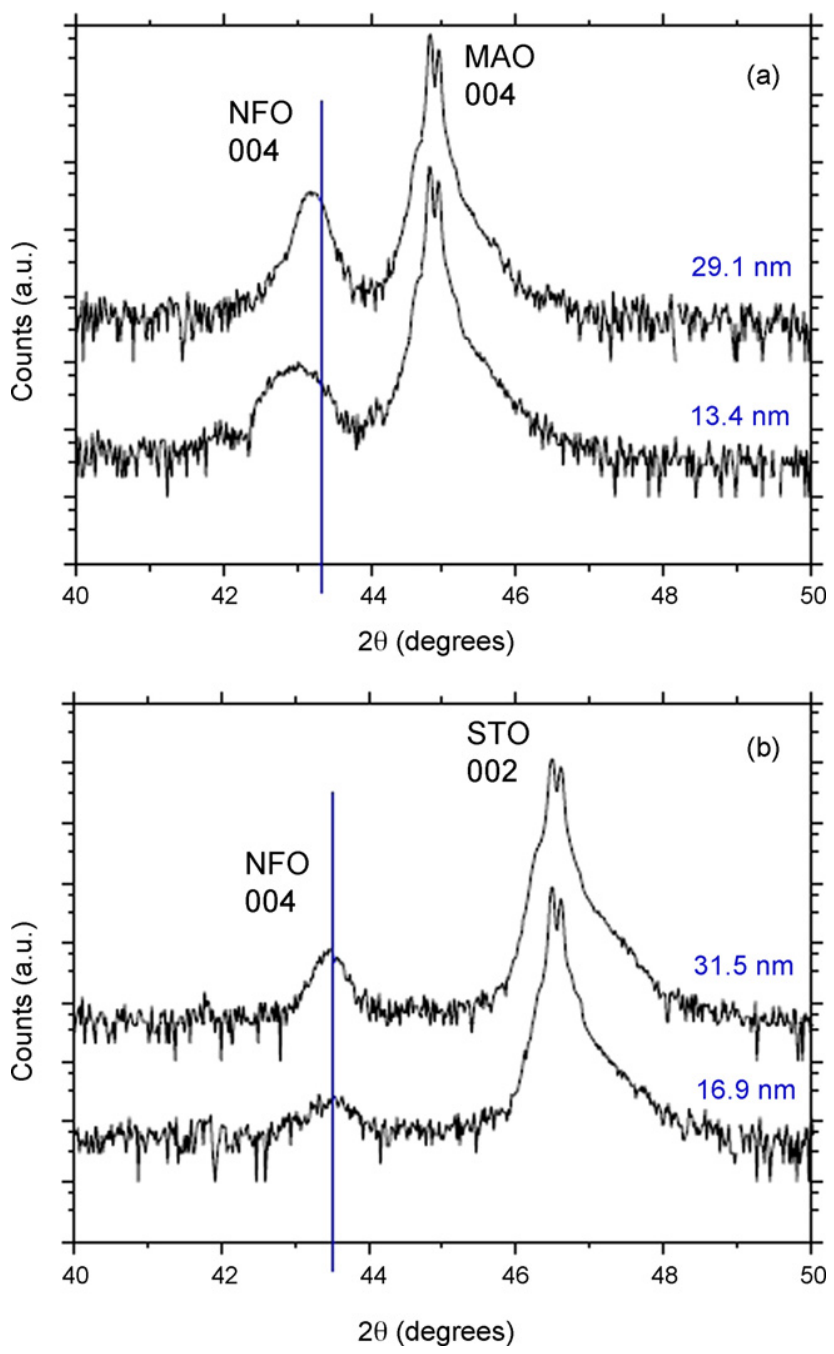

Fig. 1. XRD patterns, in configuration $\theta-2 \theta$, of NFO films over MAO (a) and STO (b) substrates, focused on the region of NFO [0 04 ] peak. Vertical lines represent the expected position for bulk single crystalline NFO $\left(43.37^{\circ}\right)$. Two different thicknesses are presented for each substrate. 
fracted region around the NFO 004 peak, which occurs near the substrate MAO 004 and STO 002 peaks. As revealed by data in Fig. 1, NFO grows (0 01 ) textured on both STO and MAO substrates. Inspection of the X-ray pattern in a broader $\theta-2 \theta$ angular range does not show any trace of other crystal orientations or phases. Detailed inspection of NFO/MAO data (Fig. 1a) reveals that the position of the 004 NFO reflection gradually shifts towards higher angles when increasing film thickness, thus indicating a gradual shrinking of the out-ofplane cell parameter which accompanies the in-plane relaxation for the cell parameters, from a strained state (compressive) occurring in the thinner films towards the bulk value. The cell parameters obtained from data in Fig. 1 are: $c=0.8384 \mathrm{~nm}$ and $c=0.8369 \mathrm{~nm}$ for $t=13.4 \mathrm{~nm}$ and $29.1 \mathrm{~nm}$ NFO films on $\mathrm{MAO}$, and $c=0.8319 \mathrm{~nm}$ both for $t=16.9 \mathrm{~nm}$ and for $31.5 \mathrm{~nm}$ NFO films on STO. The resulting room-temperature out-ofplane strain $\left.\varepsilon\left(\varepsilon=\left(c_{\text {film }}-c_{\text {bulk }}\right) / c_{\text {bulk }}\right)\right)$ amounts to $0.54 \%$ and $0.36 \%$ for NFO/MAO films and $-0.24 \%$ for both NFO/STO films.

The existence of an in-plane compressive strain in the thinner NFO/MAO films is due to the moderate mismatch parameter $f\left(f=\left(a_{\text {film }}-a_{\text {substrate }}\right) / a_{\text {substrate }}\right)$ between the structures $(3.2 \%)$ and the resulting epitaxial stress gradually relaxes when increasing film thickness. Remarkably enough, the X-ray patterns of the NFO/STO films do not show any peak-shift with thickness,

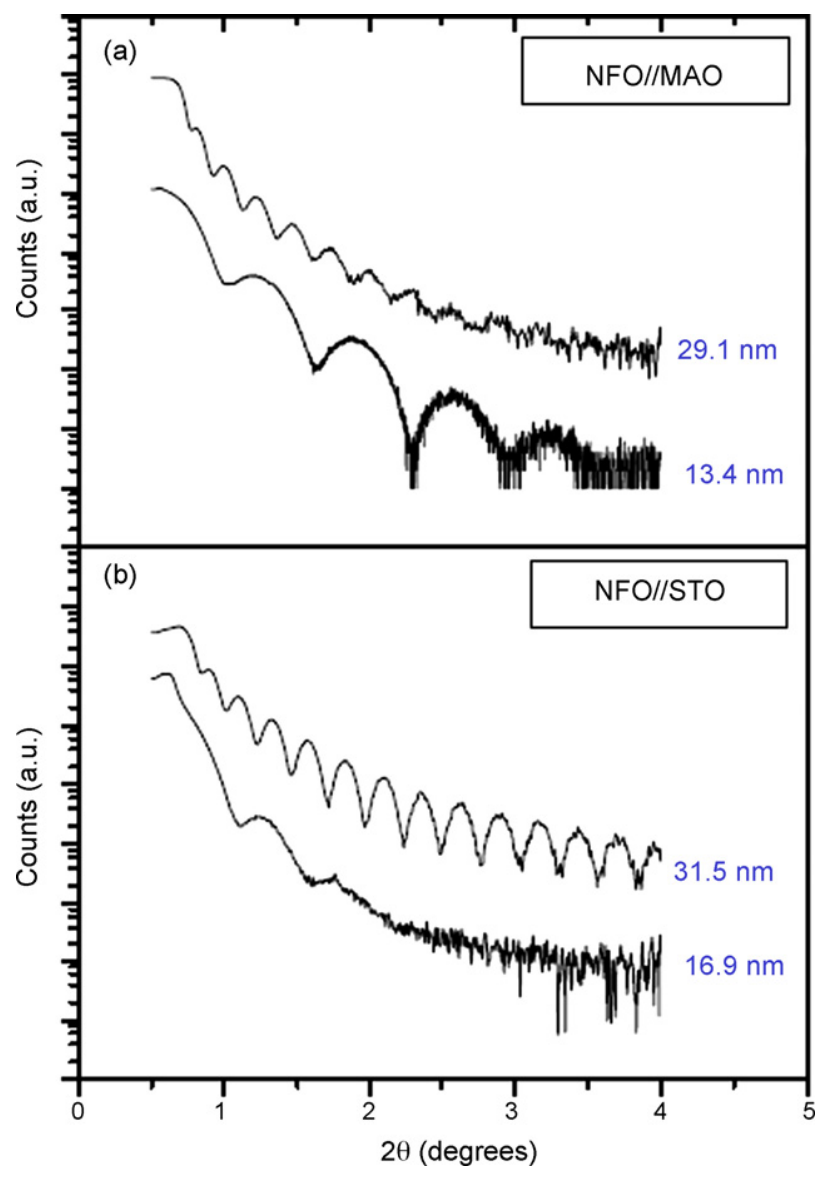

Fig. 2. XRR curves of NFO films over MAO (a) and STO (b) substrates. The measurements on the same samples presented in Fig. 1 are showed. thus suggesting a fully relaxed structure already from the thinner films. It probably results from the larger mismatch of NFO on STO ( $f=6.8 \%$, calculated for 2 unit cell of STO). Indeed the cell parameter obtained even for the thinnest film is very close to that of bulk NFO.

The XRR patterns of these films are reported in Fig. 2a (NFO/MAO) and $\mathrm{b}$ (NFO/STO). These curves give, in addition to the thickness value, an indication of the surface roughness. The thinner film on MAO presents better defined fringes, and with higher intensity than the corresponding case of STO, and this trend is found in all films grown in this thickness range; this fact can be ascribed to a larger roughness of the films grown on STO than its MAO counterpartners. Atomic force microscopy images (not shown here) also confirmed the same trend. The relatively rougher surface of NFO films on STO than in MAO reflects differences on the growth mode of NFO on these different substrates. The dissimilar lattice mismatch, but also the difference in the atomic arrangement in the $\left(\begin{array}{lll}0 & 0 & 1\end{array}\right)$ surface of spinel and perovskite can play an important role. Multicell
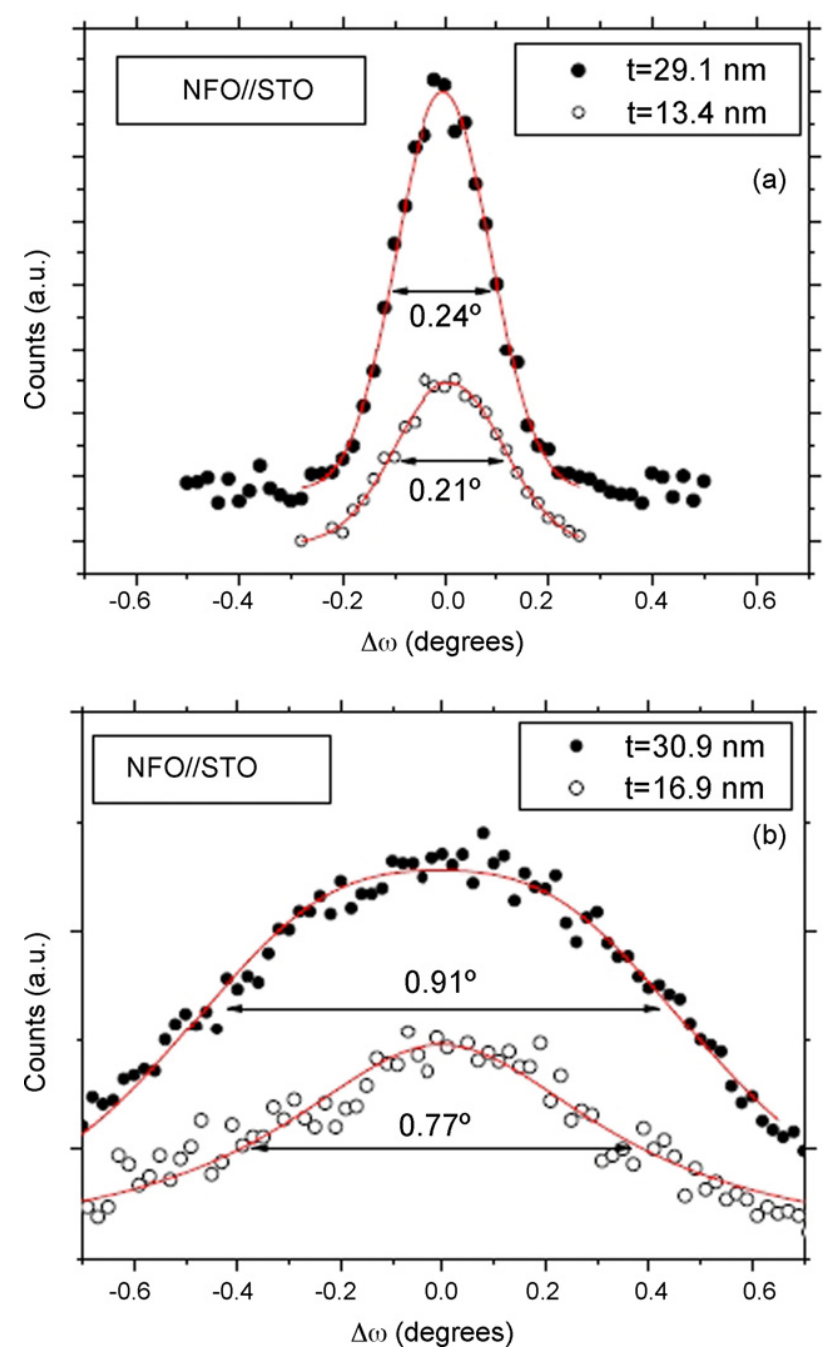

Fig. 3. Rocking curves of NFO films over MAO (a) and STO (b) substrates. Two different thicknesses are examined for each substrates; continuous line represents a Pseudo Voigt fitting of experimental data. For each curve, the full width at half maximum is reported. 
long-rage ordering is required to grow a $\left(\begin{array}{lll}0 & 0 & 1\end{array}\right)$ spinel-structure on a $\left(\begin{array}{lll}0 & 0 & 1\end{array}\right)$ perovskite one.

The differences in crystalline quality of the NFO films, depending on the substrate used, can also be inferred from the inspection of the rocking curves shown in Fig. 3a (NFO/MAO) and $\mathrm{b}$ (NFO/STO) (the red line represents a Pseudo-Voigt fit); it can be appreciated that the rocking curves of NFO/STO display a full-width at half maximum (FWHM) considerably larger than NFO/MAO films of similar thickness: for the $t \approx 31.5 \mathrm{~nm}$ NFO/STO film, FWHM $\sim 0.91^{\circ}$, while it is only $\sim 0.43^{\circ}$ for the $t \approx 29.1 \mathrm{~nm}$ NFO/MAO film. In any event, samples grown on both substrates show an increase of the FWHM with thickness, as normally expected, because the increasing of crystal size generates a high probability of defects formation.

TEM bright field images in planar view geometry are presented in Fig. 4a and c, respectively, for a film $5 \mathrm{~nm}$ thick over MAO and $6 \mathrm{~nm}$ thick over STO. No evidence of the presence of anti-phase boundaries can be seen. The corresponding diffraction patterns in Fig. $4 \mathrm{~b}$ and $\mathrm{d}$ shows the fully epitaxial character of the coatings. From these figures, and from the previously reported XRD analysis, the epitaxial relationships [ $\left[\begin{array}{lll}1 & 1 & 0\end{array}\right] \mathrm{NFO}\left(\begin{array}{lll}0 & 0 & 1\end{array}\right) / /\left[\begin{array}{lll}1 & 1 & 0\end{array}\right] \mathrm{STO}\left(\begin{array}{lll}0 & 0 & 1\end{array}\right)$ and [ $\left.\begin{array}{lll}1 & 1 & 0\end{array}\right] \mathrm{NFO}\left(\begin{array}{lll}0 & 0 & 1\end{array}\right) / /\left[\begin{array}{lll}1 & 1 & 0\end{array}\right] \mathrm{MAO}\left(\begin{array}{lll}0 & 0 & 1\end{array}\right)$ have been inferred.

Magnetic hysteresis loops of the same NFO films on MAO and STO are presented respectively in Fig. 5a and b. As a convenient reference, we include (dashed line) in these figures the saturation magnetization for NFO $\left(281 \mathrm{emu} / \mathrm{cm}^{3}\right)$ evaluated using the collinear Néel theory. Data in Fig. 5 clearly reveal that magnetization of the thinner NFO films, irrespectively on the substrate used, is substantially larger than expected. Indeed, $M_{\mathrm{S}} \approx 771 \mathrm{emu} / \mathrm{cm}^{3}$ for a $2.6 \mathrm{~nm}$ thick film on STO, and $630 \mathrm{emu} / \mathrm{cm}^{3}$ for a $5.0 \mathrm{~nm}$ thick film on MAO. It is also clear that this excess magnetization becomes progressively smaller and eventually disappears when film thickness increases. On the other hand, the thicker films present different magnetic characteristics depending on substrate material: in the case of the spinel substrate $(\mathrm{MAO})$, for $t \approx 29.1 \mathrm{~nm}$ film, $M_{\mathrm{S}} \approx 297 \mathrm{emu} / \mathrm{cm}^{3}$ thus approaching the bulk value: on the contrary, for NFO films of similar thickness $t \approx 31.5 \mathrm{~nm}$, the saturation magnetization is considerably lower than bulk NFO $\left(M_{\mathrm{s}} \approx 174 \mathrm{emu} / \mathrm{cm}^{3}\right)$.

\section{Discussion}

The spinel structure $\left(\mathrm{AB}_{2} \mathrm{O}_{4}\right)$ consists of a close-packed facecentered cubic oxygen lattice, with cations in the tetrahedral [A] and octahedral [B] interstices; these two sublattices are antiferromagnetically coupled. One unit cell contains 8 formula units. In the normal spinel structure all trivalent cations are at B-sites whereas the divalent cations occupy A-sites. Bulk $\mathrm{NiFe}_{2} \mathrm{O}_{4}$ is a totally inverse spinel structure which means that all divalent cations are at $\mathrm{B}$-sites. Therefore, $8 \mathrm{Ni}^{2+}$ ions are all found in
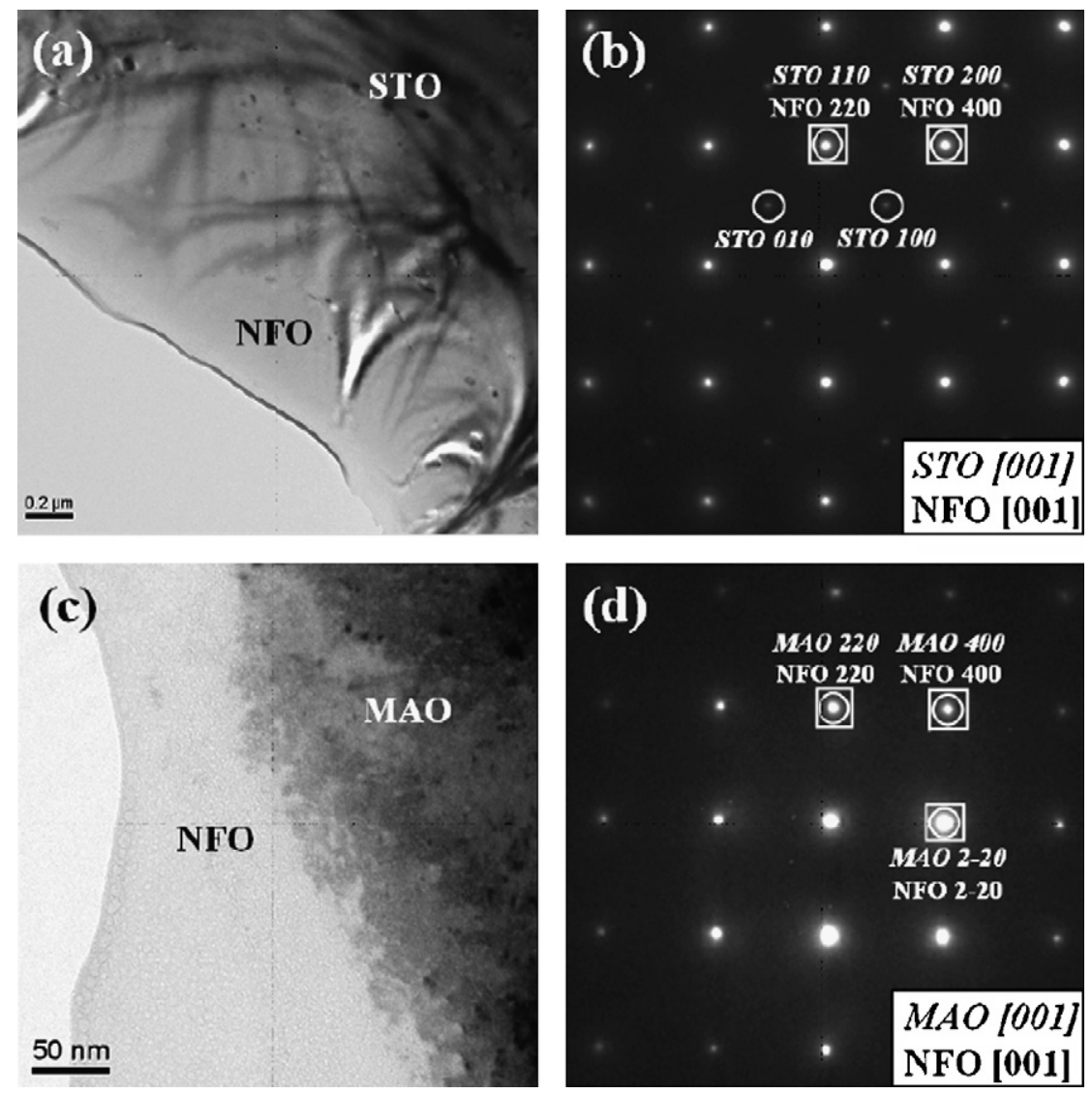

Fig. 4. TEM bright field images of NFO films over MAO (a) and STO (c) substrates and on the right side the respective diffraction pattern (b) and (d) in planar view geometry. Labels are placed on top of relative spots (italic font for substrates); a circle marks substrate reflections while a square is used for films. 

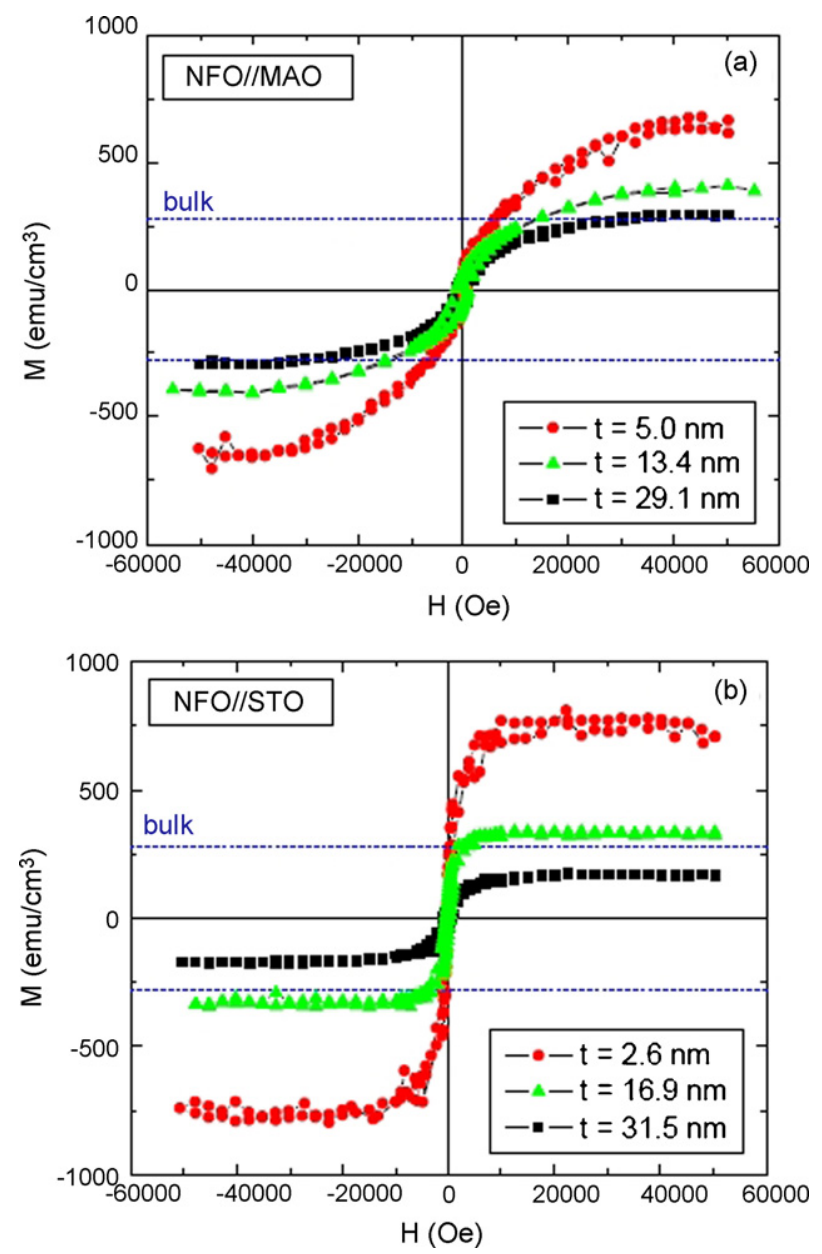

Fig. 5. Magnetic hysteresis loops of NFO films over MAO (a) and STO (b) substrates, measured with the external field parallel to the sample surface, at $10 \mathrm{~K}$. Three different thicknesses are presented; dashed lines represent the expected values for bulk single crystalline NFO $\left(\sim 281 \mathrm{emu} / \mathrm{cm}^{3}\right)$.

[B] positions, while the $16 \mathrm{Fe}^{3+}$ ions are found equally distributed over $[\mathrm{A}]$ and $[\mathrm{B}]$ sites $\left(\mathrm{Fe}[\mathrm{NiFe}] \mathrm{O}_{4}\right)$. Contributions to the total magnetic moment come from both cation types: $2 \mu_{\mathrm{B}}$ for each $\mathrm{Ni}^{2+}$ ion and $5 \mu_{\mathrm{B}}$ for each $\mathrm{Fe}^{3+}$ ion, but, while all the nickel contributions are oriented in the same direction, each iron moment is cancelled out by the presence of an equal ion with opposite magnetic orientation, because of the coupling between tetrahedral and octahedral lattices. So, the overall magnetic moment is entirely attributed to the $16 \mu_{\mathrm{B}}$ coming from the 8 $\mathrm{Ni}^{2+}$ cations for unit cell $\left(\sim 281 \mathrm{emu} / \mathrm{cm}^{3}\right)$.

As an explanation for the higher $M_{\mathrm{S}}$ observed in the thinner films, we consider the possibility for the structure to experience a transition to the normal spinel structure, where trivalent ions are only found in $[\mathrm{B}]$ sites and the divalent ones are only in [A] positions $\left(\mathrm{Ni}[\mathrm{Fe}]_{2} \mathrm{O}_{4}\right)$. In this case, the $16 \mu_{\mathrm{B}}$ for unit cell ascribed to the nickel contribution face to the $80 \mu_{\mathrm{B}}$ coming from the 16 iron ion with the opposite orientation, for a total amount of $64 \mu_{\mathrm{B}}$ for unit cell $\left(\sim 1123 \mathrm{emu} / \mathrm{cm}^{3}\right)$. This value is substantially larger than the magnetization observed for the thinner films and thus, a partial inversion $\left(\mathrm{Fe}_{1-x} \mathrm{Ni}_{x}\right)\left[\mathrm{Fe}_{1+x} \mathrm{Ni}_{x}\right] \mathrm{O}_{4}$ would account for the observed data. Venzke et al. [8] and Lüders et al. [7] invoked the same argument to account for the observation of an enhanced magnetization of ultrathin NFO films. It is worth however to mention that, so far, no other evidence has been reported. We would like to mention here that enhancement of the magnetic moment has been observed in small size ferrite particles [9] and it has been attributed to a frustration of collinear spin arrangement due to surface effects. It is not unconceivable that a similar scenario in nanometric thin films could lead to an enhanced magnetization. Spectroscopic tools able to distinguish the occupancy of the different atomic $(\mathrm{Fe}, \mathrm{Ni})$ species among the different lattice sites are required to definitely settle this question.

On the other hand, the commonly accepted explanation for the reduced $M_{\mathrm{S}}$ found for thick films over STO is the presence of anti-phase boundaries (APB) [6], which would also produce an increasing of the high-field susceptibility of the film. In that case, the extraction of the diamagnetic contributions of substrate and sample holder from the measured high-field region of the magnetization curve would include a contribution arising from the presence of APB's defects. It could be expected that the measured high-field susceptibility increases when increasing the APB's concentration. In any event, a higher concentration of APB's could be expected in samples grown on STO respect to MAO, due to the more accentuated difference between film and substrate structure, therefore, within the APB's model, one would expect a lower $M_{\mathrm{S}}$ for NFO films grown on STO than on $\mathrm{MAO}$ and a larger high-field susceptibility in NFO/STO than in NFO/MAO films. Whereas the experimental values of the saturation magnetization will support this picture, the high-field susceptibility is not found to vary significantly from film to film and thus APB's, if any, do not seem to play a role. Moreover, we were not able to individuate the presence of APB's by TEM analysis (Fig. 4a and c) of NFO films neither on STO nor on MAO and thus the origin of the reduction of magnetization of NFO films on STO remains to be elucidated. It is worth to mention that Wakiya et al. [5] analyzed the saturation magnetization of a number of spinel-oxide thin films. They noticed that mismatch values within the range $0<f<6.5 \%$ were required to obtain a full magnetization whereas for $f>6.5 \%$ a reduced magnetization was always obtained; unfortunately a microscopic description was lacking but it is remarkable that our data also fits in this picture. Before closing, however, it is worth to recall that the spinel structure forms an open network, where unoccupied sites may lead to significant atomic film/substrate interdiffusion. The eventual presence of $\mathrm{Ti}^{4+}$ cations into the NFO lattice could reduce the magnetization.

\section{Conclusions}

Epitaxial films of nickel ferrite have been grown on single crystalline $\mathrm{SrTiO}_{3}$ and $\mathrm{MgAl}_{2} \mathrm{O}_{4}$ substrates, both with a (lll 001$)$ orientation. We have shown that NFO on MAO display better crystalline quality as evidenced by narrower rocking curves and lower surface roughness. We have observed that ultrathin films ( $<20 \mathrm{~nm}$ for MAO and $<13 \mathrm{~nm}$ for STO) display a magnetization substantially larger than the bulk value. This behavior could be ascribed to formation of a partially inverse spinel structure 
during thin film growth. However, it cannot be excluded that size-effects also contribute to this consequence, by promoting a partial frustration of the antiferromagnetic coupling in the lattice. Further experiments are required to conclusively settle this question. On the other hand, thicker NFO films on MAO have a bulk-like magnetization whereas similar films on STO display a reduced magnetization. Although APB's is a common explanation for the magnetization reduction, it cannot be excluded that film/substrate cationic interdiffusion may also play a role.

\section{Acknowledgements}

Financial support by the MEC of the Spanish Government (projects NAN2004-9094-C03 and MAT2005-5656-C04) and by the European Union (project MaCoMuFi (FP6-03321) and FEDER) is acknowledged.

\section{References}

[1] U. Lüders, A. Barthélémy, M. Bibes, K. Bouzehouane, S. Fusil, E. Jacquet, J.P. Contour, J.F. Bobo, J. Fontcuberta, A. Fert, Adv. Mater. 18 (2006) 1733.

[2] U. Lüders, M. Bibes, J.F. Bobo, J. Fontcuberta, Appl. Phys. A 80 (2005) 427.

[3] H. Zheng, J. Wang, S.E. Lofland, Z. Ma, L. Mohaddes-Ardabili, T. Zhao, L. Salamanca-Riba, S.R. Shinde, S.B. Ogale, F. Bai, D. Viehland, Y. Jia, D.G. Schlom, M. Wuttig, A. Roytburd, R. Ramesh, Science 303 (2004) 661.

[4] M. Bibes, Ll. Balcells, S. Valencia, J. Fontcuberta, M. Wojcik, E. Jedryka, S. Nadolski, Phys. Rev. Lett. 87 (2001) 067210.

[5] N. Wakiya, K. Shinozaki, N. Mizutani, Appl. Phys. Lett. 85 (2004) 1199.

[6] D.T. Margulies, F.T. Parker, M.L. Rudee, F.E. Spada, J.N. Chapman, P.R. Aitchinson, A.E. Berkowitz, Phys. Rev. Lett. 79 (1997) 5163.

[7] U. Lüders, M. Bibes, J.F. Bobo, M. Cantoni, R. Bertacco, J. Fontcuberta, Phys. Rev. B 71 (2005) 134419.

[8] S. Venzke, R.B. van Dover, M. Julia, E.M. Philips, T. Gyorgy, C.-H. Siegrist, D. Chen, R.M. Werder, R.J. Fleming, E. Felder, Coleman, R. Opila, J. Mater. Res. 11 (1996) 1187.

[9] R.N. Bhowmik, R. Ranganathan, Solid State Commun. 141 (2007) 365. 